\title{
Handmade Light Absorbance Measurement Device by Using Cellophane as Filter for Educational Purpose
}

\author{
Soranut Kittipanyangam ${ }^{\mathrm{a},}$, Ponglert Rattanachinalai ${ }^{\mathrm{a}}$, Kanji Abe ${ }^{\mathrm{a}}$, Wang Lok Do ${ }^{\mathrm{a}}$, Kei Eguchi ${ }^{\mathrm{a}}$

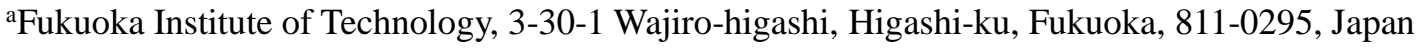 \\ *Corresponding Author: soranutac123@gmail.com
}

\begin{abstract}
In the science faculty of university, the device is not enough for every student. Therefore, this research aims to develop a light absorbance measurement device for chemical education. The students can learn about the light absorbance from the proposed device and create it. The creation is not too hard for ungraduated student. In light absorbance experiments, the kind of light is importance. To measure many solution, the proposed device must have many colors of light source. Then, the proposed device uses cellophanes as a filter for changing white light or visible light to monochromatic light. The white light consists of many color of light. When the light transmits the filter, the filter absorbs many color of light excepting filter color. Therefore, the 1 white power LED is enough for experiment. Furthermore, the color of light can be changed or mixed by color filters. To investigate properties of the proposed device, the result of it is compared with the result of the commercial spectrophotometer concerning coefficient of determination.
\end{abstract}

Keywords: Light Absorbance, UV-spectrophotometer, Beer's Law, Coefficient of Determination, Linear Regression.

\section{Introduction}

In the present, chemical has an important role in every life, for example; the medicine, food product, chemical product, and more. Those products have concentration. Therefore, the concentration of the solution is important in many fields, such as medical profession, factory, and agriculture. However, the concentration of solution cannot be measured directly. The measurement has to use many methods and convert the any value to the concentration of solution $^{(1)}$. For example, density measurement of solution by Raoult's law, this method uses the dynamic equilibrium between volatile molecules in the liquid and gas phase to calculate the density of solution ${ }^{(2)}$. Light reflection measurement, it uses the refractometer for measuring the refractive index when the light transmits the one medium to one medium. The refraction of the light is measured and calculated the concentration of solution ${ }^{(3)}$. Light absorption measurement, this measurement uses the ratio of value of light, which transmits the solution, to calculate the concentration of solution $^{(1)}$.

The light absorption is generally employed in analytical chemistry laboratories. It uses the UV-spectrophotometer which is a light absorbance measurement device. The UVspectrophotometer uses any wavelength of light, which is monochromatic light, to measure the solution. However, the UV-spectrophotometer has very high price. Therefore, the faculty of university which has many students cannot purchase the light absorbance device for every student. For this reason, there are many researches about the spectrophotometer. For example, first research is the development of a portable spectrophotometer for noncontact color measurement. This research utilizes the grating to diffract the visible light to rainbow light and then the diffracted light is used to transmit the solution ${ }^{(4)}$. After the transmitted light is measured by a photodiode array as a light detector. The photodiode array has many diodes. Therefore, it can detect all colors of light diffracted from grating. Second, Bano's spectrophotometer applies the compact disc as diffraction grid for making the color light ${ }^{(5)}$. This research uses the slit for blocking other colors of light, because the diffraction of visible light creates the rainbow light. The light absorbance measurement must use the monochromatic light. Next research is a low cost LED based spectrometer used the many LED as light source. Each LED has different color of light which replaces with the light diffraction ${ }^{(6)}$. From abovementioned researches, the color of the light source is 
important in the light absorbance measurement. Moreover, the device is huge and heavy. For this reason, it is not convenient to use in a chemical class.

To support chemical education, we develop a light absorbance device which is convenient for students. The proposed device uses the color filter for making the monochromatic light. The color filter is crated by the cardboard and cellophane. The number of filters are made following the colors of cellophane. When the proposed device has many colors of filter, the proposed device can measure many color of solution.

Furthermore, 2-3 filters can be used together for making other color or decreasing the light intensity. The proposed handmade device is smaller and cheaper than commercial spectrophotometers and has portability. Furthermore, to increase the efficiency of education, the data collection of the proposed device uses a wireless network. It is created by the interface unit Xbee which is assembled in the proposed device. The interface Xbee sends the data to a computer in text file, and then the text file is collected in Microsoft Excel. The program in Microsoft Excel is developed by visual basic.

\section{Principle of analysis}

\subsection{Light absorbance}

The light absorbance occurs when the light transmits some transparent materials. The transparent material absorbs one part of the light ${ }^{(1)}$. In chemical analysis, the light absorbance is calculated by the common logarithm of the ratio of incident to transmit radiant light though a solution. Fig. 1 illustrates the absorption of solution. When the incident light goes through the sample, the transmitted light intensity remains $70 \%$ of the incident light intensity. The solution absorbs the light intensity $30 \%$.

Although the solution absorbs the light, the solution absorbs each color of light differently. The white light consists of many colors of light. When the white light goes though the solution, the solution absorbs all colors of light in different values. The color of light which transmits the solution is color of solution. For this reason, the color of solution has specific light absorbance. The opaque material is also same case. The visible light falls on the opaque material. The opaque material absorbs the color in the visible light which each color is absorbed in different value. The light reflected from the opaque material to the eyes is a color of material. The relation between the color of solution, wavelength period and color of light, which is absorbed best, is shown in Table1. For example, green solution can absorb purple light (680-780nm) best, orange solution can absorb blue light (440-470 $\mathrm{nm})$ best.

\subsection{Beer's law}

Beer's law or Beer-Lambert law explains about attenuation of light. First, the light absorbance is common logarithm of the ratio of incident to transmit radiant light though material in Eq. (1). In Eq. (1), $A$ is absorbance, $I_{0}$ is incident light intensity and $I$ is transmitted light intensity ${ }^{(7)}$.

$$
A=\log \frac{I_{0}}{I}
$$

Second, the light absorbance is direct variation with the molar absorption coefficient, concentration of solution, and the path length of the sample in Eq. (2). Therefore, the concentration of solution can be found by Eq. (2).

$$
A=\varepsilon c l
$$

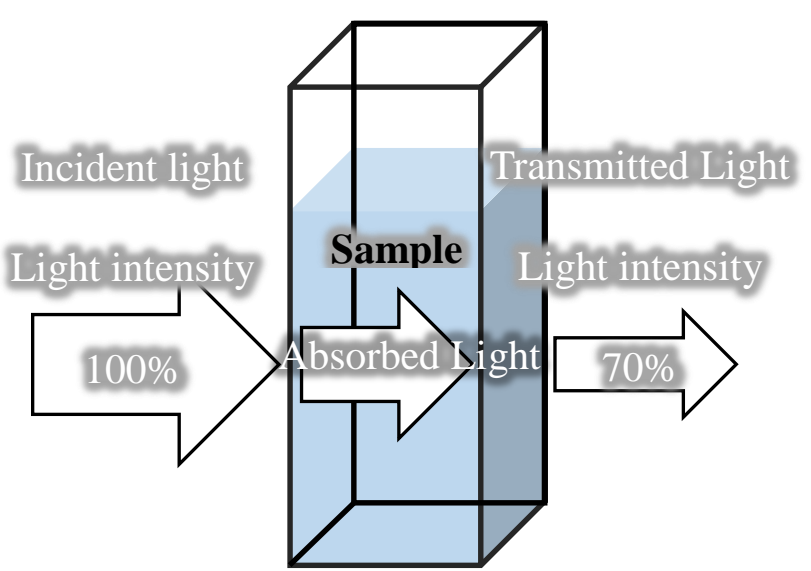

Fig. 1. Light absorbance.

Table 1. Light absorbance.

\begin{tabular}{|l|l|l|}
\hline Wavelength & Absorbance color & Color of solution \\
\hline $380-420$ & Violet & Green-yellow \\
\hline $420-440$ & Violet-blue & Yellow \\
\hline $440-470$ & Blue & Orange \\
\hline $470-500$ & Blue-green & Red \\
\hline $500-520$ & Green & Purple \\
\hline $520-550$ & Yellow-green & Violet \\
\hline $550-580$ & Yellow & Violet-blue \\
\hline $580-620$ & Orange & Blue \\
\hline $620-680$ & Red & Blue-green \\
\hline $680-780$ & Purple & Green \\
\hline
\end{tabular}




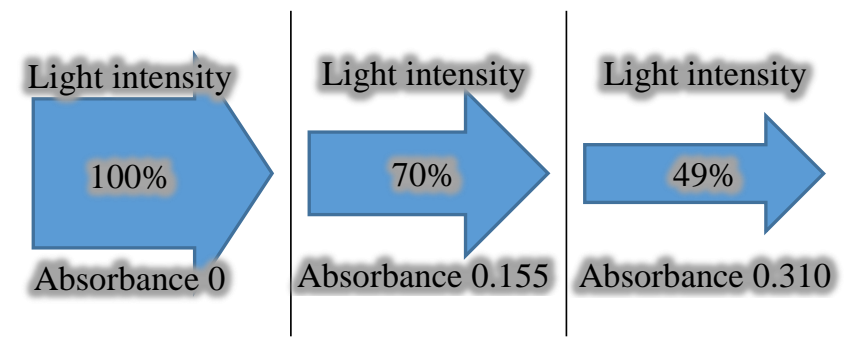

Fig. 2. Absorption of each layer of medium.

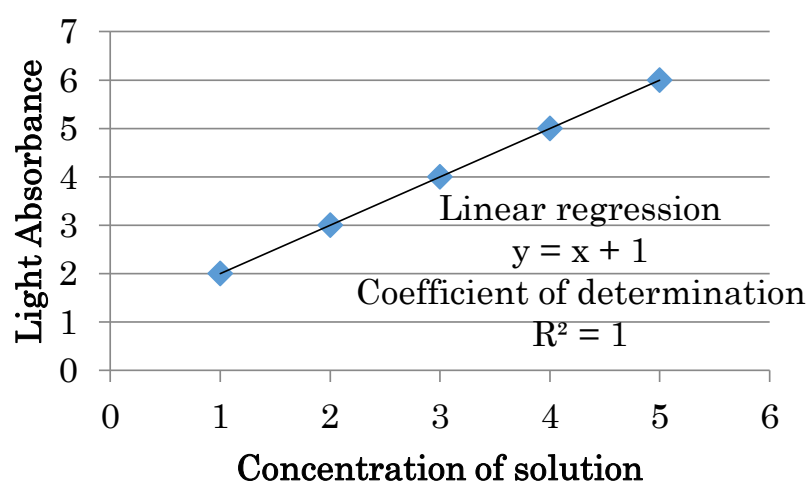

Fig. 3. Calibration curve.

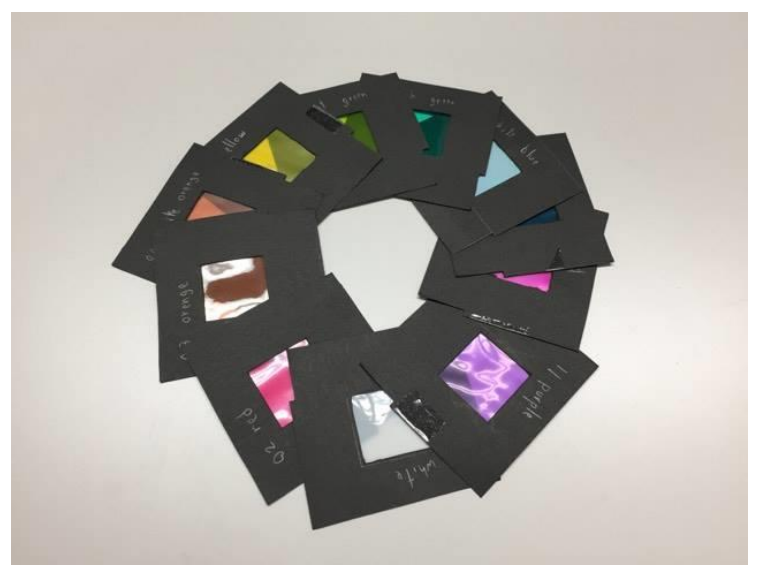

Fig. 4. Cellophane filter.

Table 2. List of color filters.

\begin{tabular}{|c|c|}
\hline Filter number & Color of filter \\
\hline 1 & White \\
\hline 2 & Red \\
\hline 3 & Orange \\
\hline 4 & Turbid orange \\
\hline 5 & Yellow \\
\hline 6 & Light Green \\
\hline 7 & Dark green \\
\hline 8 & Turbid blue \\
\hline 9 & Blue \\
\hline 10 & Violet \\
\hline 11 & Purple \\
\hline
\end{tabular}

Third, when the monochromatic light transmits the homogenous medium, each layer of medium, which the path length of each layer is equal and same material, absorbs the proportion of the light intensity. Fig. 2 shows the absorption of each layer of medium. Each layer absorbs the light intensity $30 \%$. The first incident light intensity is $100 \%$. The light is not absorbed yet, the light absorbance is 0 . When the light transmits the first layer, the light intensity remains $70 \%$ and the light absorbance increases to 0.155 . The light absorbance is calculated by Eq. (1). When the remained light transmits the second layer, the light intensity remains $49 \%$ from $70 \%$. The absorbance increases to 0.310 when the remained light compares with the first incident light intensity. If the light intensity, which transmits the second layer, is compared with the light intensity transmitting the first layer, the light absorbance is 0.155 . It is same the light absorbance which transmits the first layer, because each layer absorbs the light in same value.

\subsection{Coefficient of Determination}

The coefficient of determination is a number that shows how well relation of statistical model ${ }^{(8)}$. It can predict the data coming in the future or other related information in statistics. It denotes $R^{2}$ (the square of the correlation coefficient) in Eq. (3).

$$
R^{2}=\left\{\frac{\sum_{i=1}^{n}\left(x_{i}-\bar{x}\right)\left(y_{i}-\bar{y}\right)}{\sqrt{\sum_{i=1}^{n}\left(x_{i}-\bar{x}\right)^{2} \sum_{i=1}^{n}\left(y_{i}-\bar{y}\right)^{2}}}\right\}^{2}
$$

The value of the coefficient of determination is during 0 to 1 . If the coefficient of determination is high, it shows the good relation of each data. Fig. 3 shows the ideal coefficient of determination that value is 1 .

\subsection{Linear Regression}

The linear regression is an approach for modeling the relation between a scalar dependent variable $Y$ and one or more explanatory variable (or independent variable) denote by $X$ in Eq. $(4)^{(8)} . b$ is slope and $a$ is constant when $\mathrm{X}$ is 0 . The ideal linear regression is shown in Fig. 3.

$$
Y=a+b X
$$

\subsection{Calibration curve}

Calibration curve is a graph explaining the relation between variable $Y$ and variable $X$ from the linear regression ${ }^{(8)}$. Therefore, the calibration curve is plotted from the linear regression. The line in the calibration curve is always linear. Fig. 3 shows the ideal calibration curve which has linear regression and coefficient of determination. 


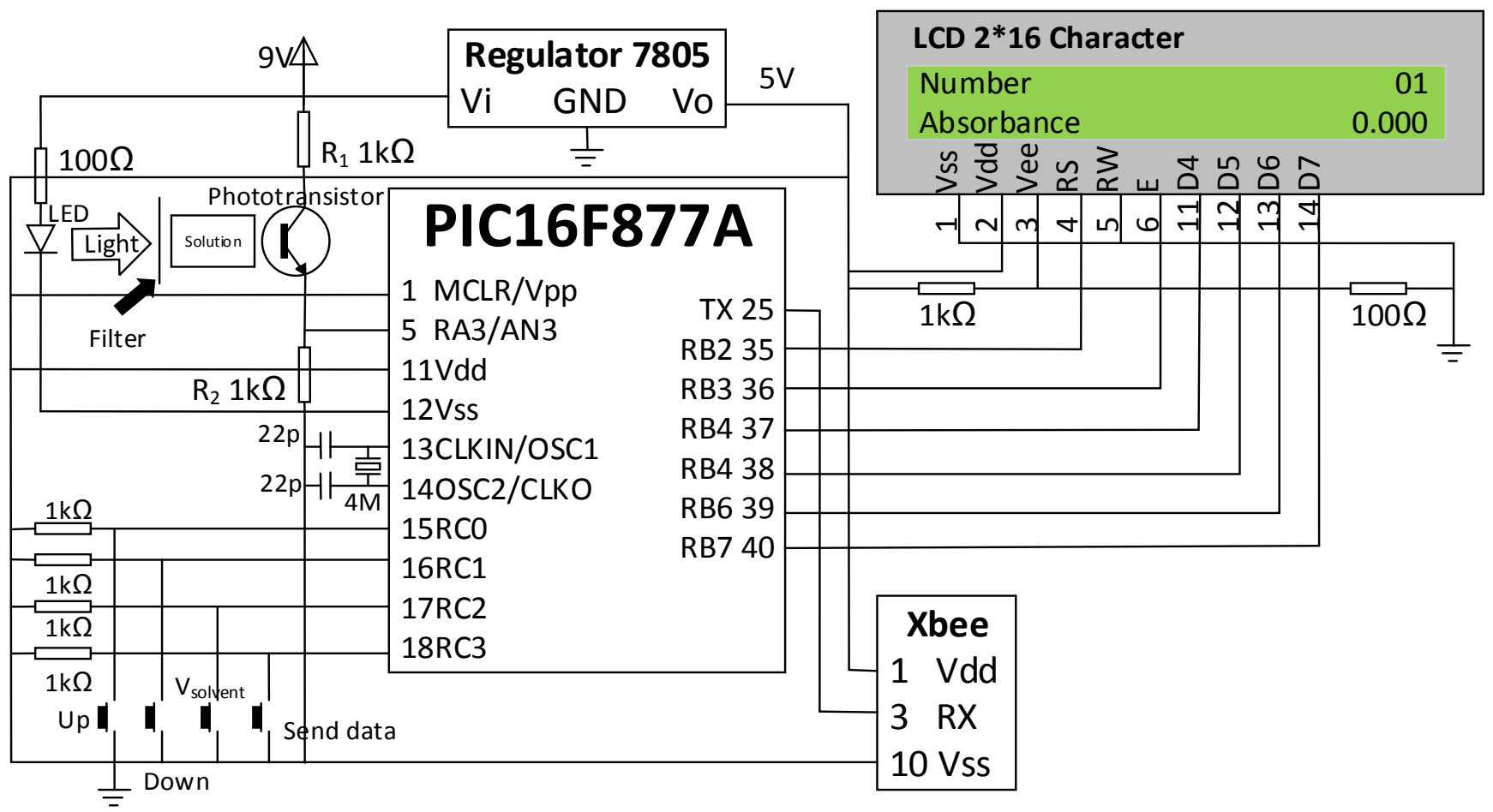

Fig. 5. Circuit configuration.

\section{Proposed device}

\subsection{Light source}

The key point of the light absorbance measurement is light source following the Beer's law. The light source has to be monochromatic light. As discussed in introduction part, there are many methods making the monochromatic light. This research uses the power LED $9 \mathrm{~W}$ as light source and the filters to convert the visible light to monochromatic light shown in Fig. 4. The filters are made from paperboard and cellophanes. The filters has 11 colors. The list of color filters and filter number are shown in Table 2. Furthermore, the filter does not only change the white light to monochromatic light, but also it decreases the light intensity. In the case of the low concentration measurement, the light source must be low brightness because the transmitted light is too much for the detector. Therefore, 2-3 filter are used. Furthermore, the use of more than one filter, it changes the color of light to other color. The proposed device can measure more solution. However, the light cannot transmit the filter, if the filters are used too much or the very dark filter is used.

\subsection{Circuit configuration}

The circuit configuration of the proposed device is shown in Fig. 5. The power supply is battery 9V. It is used to turn on the power LED, divided to the phototransistor as input voltage, and converted to $5 \mathrm{~V}$ by regulator 7805 for using the microcontroller, LCD and interface. The light absorbance is calculated by microcontroller. It calculates by the input voltage at pin 5 which is dropped on $\mathrm{R}_{2}$. This voltage is controlled by phototransistor following light intensity. The light absorbance is shown on the LCD which connects with the port B of microcontroller. The LCD $2 * 16$ character shows the device number and the light absorbance. The device number is employed for listing the data sending to a computer by Xbee. The Xbee connects with pin TX of microcontroller. The number device can be changed by the up button and down button. The send data button is utilized to send data to a computer. The $\mathrm{V}_{\text {solvent }}$ button is used for calculating the light absorbance which it will be described in calculation topic.

\subsection{Measurement}

The measurement of the proposed device utilizes the white power LED as light source and the phototransistor as light detector. To measure the solution, the light source is monochromatic light following the beer's law. The filters shown in Fig. 3 are used for changing the visible light to the monochromatic light. The proposed device utilizes input voltage dropped on $\mathrm{R}_{2}$ for calculating the light absorbance. The voltage from the electric source is divided by the half 
voltage divider $R_{1}$ and $R_{2}$. Therefore, the maximum voltage doesn't exceed 4.5V. The phototransistor controls the input voltage dropped on $\mathrm{R}_{2}$ following the light intensity which transmitting the solution during $0-4.5 \mathrm{~V}$. When the detector receives the light too much, it decreases its resistance. The minimum is $0 \Omega$ and the voltage on $R_{2}$ is 4.5 . When there is not light falling on the detector, the resistance is infinite. The voltage dropped on $R_{2}$ is 0 .

\subsection{Calculation}

The calculation of the light absorbance measurement must use the incident light intensity and transmitted light intensity following (1). Therefore, the device must be assembled by 2 detectors for measuring. To decrease the cost by assembling only 1 detector, the light measurement is converted by measuring the light intensity transmitting the sample only. The incident light is converted to light intensity when the light transmits solvent $\left(I_{\text {solvent }}\right)$ and the transmitted light intensity is converted to light intensity when the light transmits the solution $\left(I_{\text {sample }}\right)$ in (5). Moreover, the detector gives the resistance information only. Although, the electric circuit can convert the resistance to the voltage, there are not light intensity. Therefore, the light intensity in equation must be converted to voltage following (5). $V_{\text {solvent }}$ is voltage when the light transmits solvent and $V_{\text {sample }}$ is voltage when the light transmits the solution. Moreover, to subtract the noise falling into the detector, the constant value $\left(V_{\text {zero }}\right)$ is add into the equation following (5). The absorbance is obtained by substituting (1) and (5) into (6).

$$
\begin{aligned}
& \frac{I}{I_{0}}=\frac{I_{\text {sample }}}{I_{\text {solvent }}}=\frac{V_{\text {sample }}-V_{\text {zero }}}{V_{\text {solvent }}-V_{\text {zero }}} \\
& A=-\log \frac{V_{\text {sample }}-V_{\text {zero }}}{V_{\text {solvent }}-V_{\text {zero }}}
\end{aligned}
$$

In (6), the voltage is measured. Therefore, the program is modified from the voltage meter program ${ }^{(9)}$. The microcontroller receives the voltage during $0 \mathrm{~V}-5 \mathrm{~V}$ and converts it by A/D to 10 bit binary digit during $0-1023$. To revert the binary digit to voltage value, the binary digit is multiplied with 0.489 to voltage value. The program represents that voltage into the $\mathrm{V}_{\text {sample }}$ and calculates the light absorbance in real time. The $\mathrm{V}_{\text {solvent }}$ be represented by the voltage of $\mathrm{V}_{\text {sample }}$, when the $\mathrm{V}_{\text {solvent }}$ is pushed. Hence, the light absorbance shown in the LCD is 0 , because of the equal between the $\mathrm{V}_{\text {sample }}$ and $\mathrm{V}_{\text {solvent. The light absorbance is }}$ calculated in proposed device during $0-2$. If the light cannot transmit the filter, the light shown in the LCD is 2.000 . When $\mathrm{V}_{\text {solvent }}$ is memorized in this case, the light absorbance will be 2.618. It shows that the device cannot measure it.
The coefficient of determination is used for observing the relation between the light absorbance and the concentration of solution. In (3), $x$ is concentration of solution and $y$ is light absorbance. The linear regression is utilized for calculating the unknown concentration of solution. It uses cross-multiplication to find the unknown light absorbance and the concentration of solution. The variable is same with coefficient of determination. In (4), $a$ is constant when the concentration is 0 .

\subsection{Data collection}

The data sent from the microcontroller is the device number and the light absorbance. The proposed device sends data by USART asynchronous transmitting. The sent data is text file. The one time sending data has 7 character. The first two is device number and the others is light absorbance. For example, in case of 010.123 , the device number is 1 and the light absorbance is 0.123 . The device number decides the column which lists the light absorbance in Microsoft Excel.

The interface assembled in the proposed device is Xbee. It can create the wireless network which can send the data in the network easily. The Xbee can create the many type of network. This research chooses the mesh network. This network is illustrated in Fig. 6. In this network, there are 1 coordinator as master device which connects with the computer and the others are router as slave device which is assembled in the proposed device. The router device can send data to the coordinator directly or through the others Xbee in the network to the master device. Then, the master device send the data to a computer. For this reason, if the one slave device is lost, the others can be replaced with the lost device.

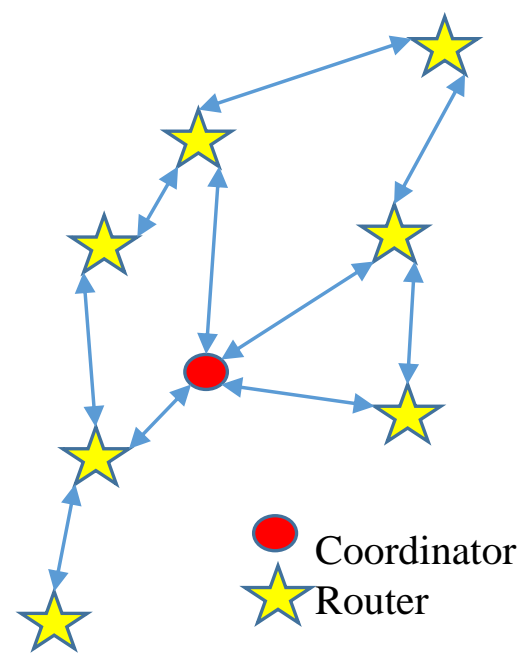

Fig. 6. Wireless network mesh type 
The program receives the text file to collect the data is Microsoft Excel. It is developed by the visual basic. The program receives the measured file and then the program lists the data into each column. Furthermore, the program can create the calibration curve like Fig. 3 which shows the coefficient of determination and the linear regression. Moreover, it can create the comparison result of each device number like Fig 7, 8.

\section{Experimental result}

The experiment measures the 3 colors of solution, red, blue, and green which are main colors of light. Each solution has 5-6 concentration. If the experiment measures more concentration, the coefficient of determination is more accurate. The preparation of concentration of solution uses the commercial spectrophotometer by the light, which is absorbed by each solution best. Therefore, the coefficient of determination is close to 1 or 1 . To check the efficiency of the proposed device, the coefficient of determination is used for comparing with the commercial spectrophotometer. The proposed device uses the light, which is absorbed by concentration best, being like the commercial spectrophotometer. The result from the best light gives the light absorbance which each value is apart. Therefore, the observation of error is the easiest. Furthermore, using the best light, it is the highest efficiency for checking the coefficient of determination.

\subsection{Red solution}

The first experimental solution is the red solution. From the table. 1, the red solution absorbs the blue-green light (470-500nm) best. Fig. 7 illustrates the light absorbance graph measured from the commercial spectrophotometer. It shows that the red solution absorbs about 500nm light best. The result of proposed device is shown in Fig. 8. The filter is used in the experiment shown in table 2 . The best absorbed light is dark green that filter number is 7 . The result is shown in table 1 . In the case of the red solution, the comparison result is shown in Fig. 9. The coefficient of determination of the commercial spectrophotometer is 0.9972 . On the other hand, the coefficient of determination of the proposed device is 0.9972 . The efficiency of the proposed device which coefficient of determination of proposed device compares with that of the commercial spectrophotometer is $100 \%$ and the error is 0 .

\subsection{Blue solution}

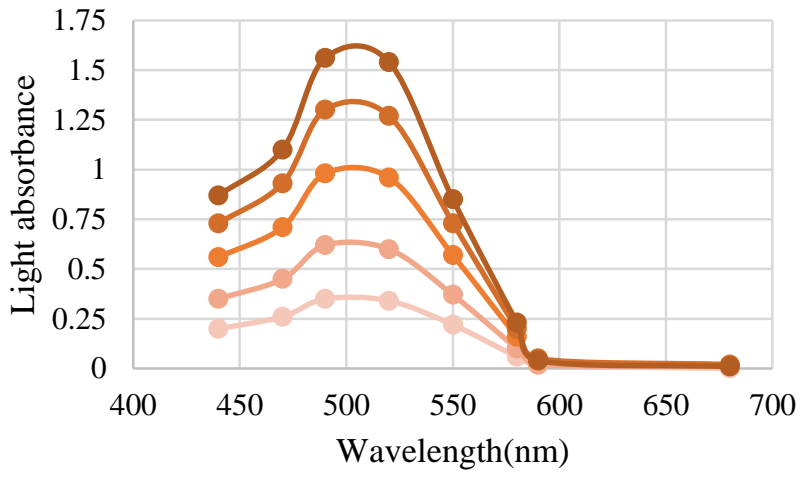

Fig. 7. Red solution result measured from the spectrophotometer.

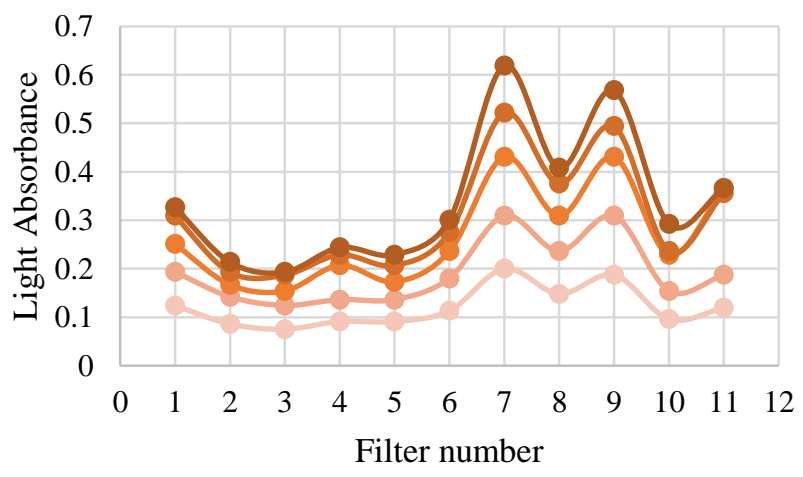

Fig. 8. Red solution result measured from the proposed device.

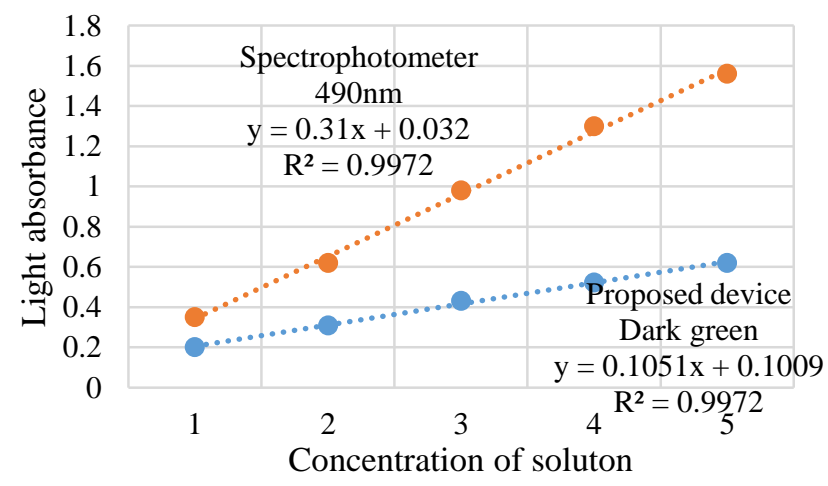

Fig. 9. Comparison result in the case of red solution.

The second experiment solution is the blue solution. The blue solution absorbs orange light (580-620 nm) best shown table. 1. The blue solution result measured from the spectrophotometer is illustrated in Fig. 10. It shows that the blue solution absorbs about $600 \mathrm{~nm}$ light best. The result of the proposed device is shown in Fig. 11. The best absorbed light is orange that filter number is 3 .

Fig. 12 illustrates the comparison result in the case of blue solution. The coefficient of determination of spectrophotometer is 1 and that of the proposed device is 0.9924. The efficiency of the proposed device which coefficient of determination of proposed device compares 
with that of the commercial spectrophotometer is $99.24 \%$ and the error is $0.76 \%$.

\subsection{Green solution}

The last experimental solution is green solution. The green solution absorbs the purple light about (680-780nm) best shown in table. 1 . The green solution result measured from the commercial spectrophotometer is shown in Fig. 13. The green solution absorbs about $440 \mathrm{~nm}$ light best which the color of light is violet-blue light. The result does not follow with the table. 1 . In table.1, the green solution absorbs 3 colors of solution best, violet light (380-420nm), red light (620-680 nm), and purple light (680-780 nm).

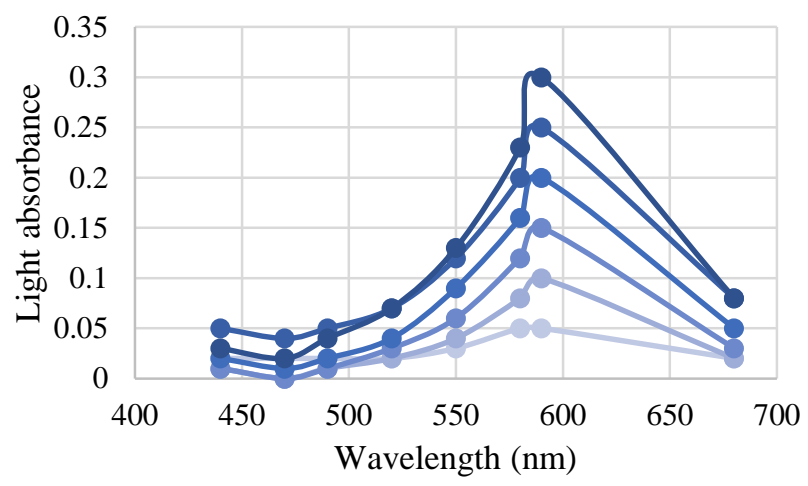

Fig. 10. Blue solution result measured from the spectrophotometer.

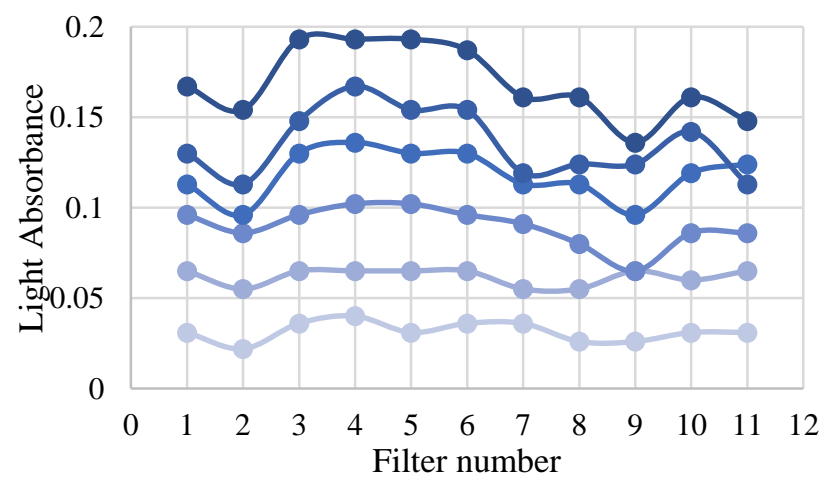

Fig. 11. Blue solution result measured from the proposed device.

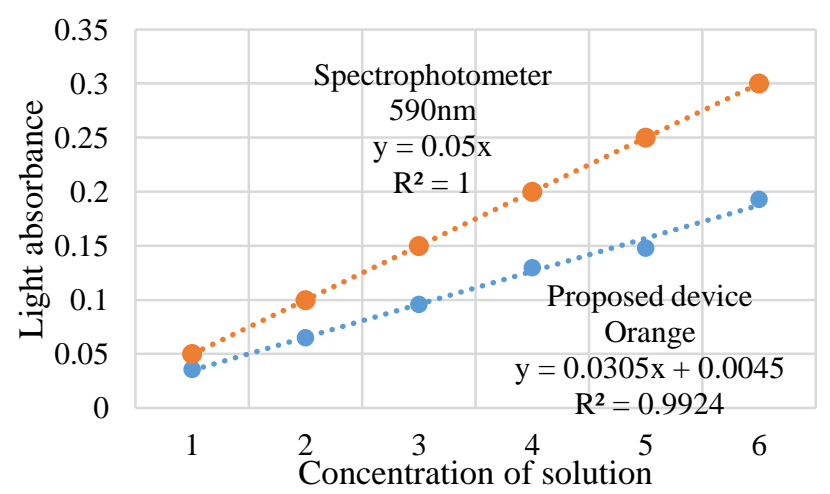

Fig. 12. Comparison result in the case of blue solution.

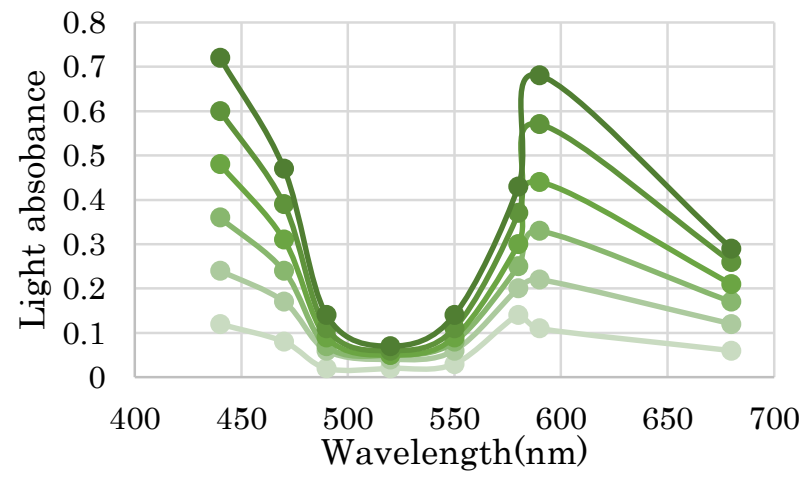

Fig. 13. Green solution result measured from the spectrophotometer.

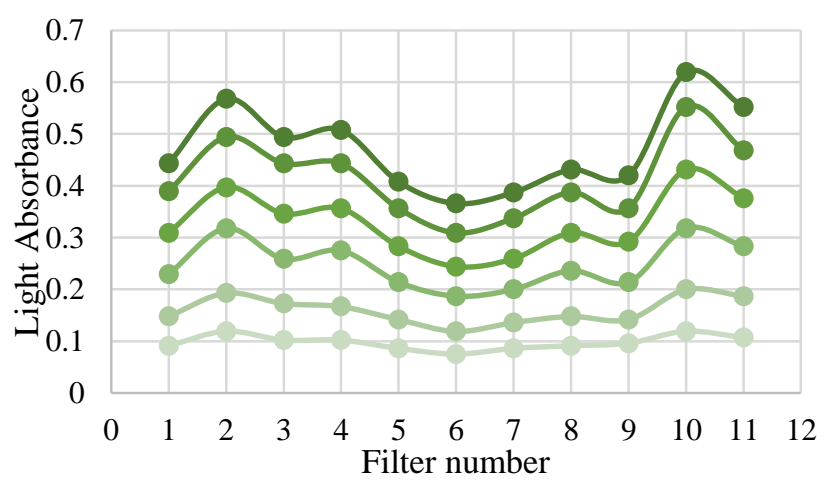

Fig. 14. Green solution result measured from the proposed device.

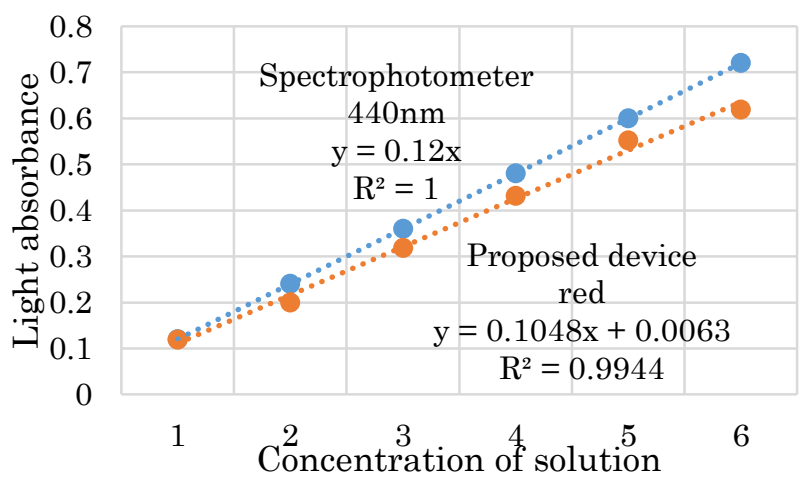

Fig. 15. Comparison result in the case of green solution.

However, the light absorbance of less than $500 \mathrm{~nm}$ light in fig. 13 is increasing. However, the compared commercial spectrophotometer doesn't have less than $440 \mathrm{~nm}$ light source. If the commercial device has 380-420nm light source, the best absorbed light may be in this period. The green result measured from the proposed device is illustrated in Fig. 14. The best absorbed light is red that filter number is 10 . The comparison result in the case of green solution is shown in Fig 15. The efficiency of the proposed device which coefficient of determination of proposed device compares with that of the commercial spectrophotometer is $99.44 \%$ and the error is $0.54 \%$. 


\section{Discussion}

The handmade light absorbance device using a cellophane filter was developed in this research. In experiments, the result of the proposed device result was compared with that of the commercial spectrophotometer. The conventional spectrophotometer has a light source which is known the wavelength. However, the light source of the proposed device is known only the color. In this point, the commercial spectrophotometer is useful for the scientists who want the detailed information. Furthermore, the color light, which doesn't know the wavelength, is not serious problem, because in education the student of the science faculty has to know about relation between the color of light and the wavelength.

In the class, there are 1-2 spectrophotometer only which are not enough for every students. The student cannot use it for a long time. In the study, the students experiment with the spectrophotometer a few time. They cannot understand the measurement of the solution. Since the proposed device is cheap, the science faculty can purchase the proposed device in bulk. Every students can experiment for a long time. The most of student exercises are the calculation of the unknown concentration. When many known concentration solutions are measured, the calibration curve and the linear regression are calculated. To find the unknown concentration, the light absorbance of the unknown concentration is replaced in the linear regression. For example, in the result of the green solution from the spectrophotometer in Fig. 15, the linear regression is $y=0.12 x$. If the light absorbance is 0.72 , the concentration is 6 .

\section{Conclusions}

The handmade light absorbance device using a cellophane filter has been proposed in this paper. The monochromatic light source is made by the color filter. It solves the cost problem of the spectrophotometer by assembling the low cost and portable handmade device. The system of the proposed device is not complicated.

To replace the spectrophotometer in education, the professor of faculty of science requires the coefficient of determination of the proposed device more than 0.99XX. It means the efficiency of the proposed device is more than $99 \%$ when the coefficient of determination of the proposed device compares with that of the spectrophotometer. From the result in experiments concerning the 3 solution red, blue, and green, the efficiency of the proposed device when the coefficient of determination compares with that of the commercial spectrophotometer is $100 \%$ of red solution result, $99.24 \%$ of blue solution result and $99.44 \%$ of the green solution result. Therefore, the proposed device can be replaced with the commercial spectrophotometer in the education.

The detector of the proposed device has response with about $800-1300 \mathrm{~nm}$ (red, purple) light best. Therefore, the slop in the result measured by red light is high. The calculated concentration becomes more accurate than low slop result. In a future study, to increase the efficiency of the calculation of concentration, we would like to match the color of light with the response of the detector. If the match between the light source and the light source is good compatibility, the change of the voltage is higher than before. Each light absorbance is more extended than before. Therefore, the slope becomes higher.

\section{References}

(1) C. Areejitranusorn "Absorption spectrophotometer", Scientific instrument, pp.227-271, 2001

(2) T. Luangvaranunt "Behavior of solution", Thermodynamics for metallurgical and materials engineering, pp.112-129, 2014

(3) L. Xu, S. Zhang, Y. Tan, S. Zhang, and L. Sun, Member, IEEE "Refractive Index Measurement of Liquids by Double-Beam Laser Frequency-Shift Feedback", IEEE Photonics Technology Letters, Vol. 28, No.10, pp.10491052, 2016

(4) S.M. Lui, "The Development of a Portable Spectrophotometer for Noncontact Color Measurement", IEEE Transactions on instrumentation and measurement, Vol. 53, No. 1, pp.155-162, 2004

(5) S. Bano, T. Altaf, and S. Akbar, "Microcontrolled Based Spectrophotometer Using Compact Disc As Diffraction Grid”, IEEE, pp.332-336, 2010

(6) T. Yeh and S. Tseng, "A Low Cost LED Based Spectrometer", Jornal of the Chinese Chemical Society, Vol.53, pp.1067-1072, 2006

(7) S. J. Tavener and J. E. Thomas-oates, "Build your own spectrophotometer", EDUCATION IN CHEMISTRY, pp.151-154,2007

(8) M. Tiantong, "Regression Analysis", Statistics and Research Methods in Information Technology, pp.363386,2005

(9) Smart learning team, "Digital voltage meter project", Advance PIC Microcontroller in C, pp.406-410, 2012 